\title{
A Multi-branch Linear Amplification with Nonlinear Components Technique
}

\author{
João Guerreiro ${ }^{(1,2)}$, Rui Dinis ${ }^{(1,3)}$, Paulo Montezuma ${ }^{(1,3)}$, João P. Oliveira ${ }^{(3)}$, Mário $\operatorname{Silva}^{(1,2)}$ \\ (1) IT, Instituto de Telecomunicações, Lisboa, Portugal. \\ (2) UAL, Universidade Autónoma de Lisboa, Lisboa, Portugal. \\ (3) FCT, Universidade Nova de Lisboa, Monte de Caparica, Portugal.
}

\begin{abstract}
Single carrier modulations with high order constellations or orthogonal frequency division multiplexing (OFDM) modulations can be employed to obtain spectrally efficient communications. However, such modulations lead to signals with large envelope variations, where the amplification is challenging and usually energy-inefficient. The linear amplification with nonlinear components (LINC) makes use of two efficient, nonlinear power amplifiers for an efficient amplification. In this work, we propose the multi-branch LINC (MB-LINC) technique, which allows the use of highly-efficient nonlinear amplifiers without increasing the nonlinear distortion effects, even if the amplifiers are not balanced ${ }^{1}$.
\end{abstract}

Index Terms-OFDM, PAPR, amplification, energy efficiency.

\section{INTRODUCTION}

Two of the most important aspects of wireless communication systems are the energy and the spectral efficiency. Achieving these two goals simultaneously is a very challenging task, specially when considering spectrally efficient modulations such as single carrier modulations with high order constellations or orthogonal frequency division multiplexing (OFDM) modulations [1], which are known to exhibit large peak-toaverage power ratio (PAPR). To avoid nonlinear distortion effects, which are more critical for larger constellations, and to avoid out-of-band emissions and maintain the high spectral efficiency of such modulations, one should consider quasilinear power amplifiers (PA). However, these amplifiers have low amplification efficiency and require high output back-off to accommodate the high PAPR of the input signals in the linear amplification region, which reduces further more the energy efficiency. Thorough the last years, many techniques that aim to reduce the PAPR prior to the amplification and improve the amplification efficiency have been proposed [2], [3]. Besides these PAPR reducing techniques, another solution for the amplification issues associated with spectral efficient transmission schemes is the linear amplification with nonlinear components (LINC) technique [4], where two simple and

\footnotetext{
${ }^{1}$ This work is supported by the European Regional Development Fund (FEDER), through the Competitiveness and Internationalization Operational Program (COMPETE 2020) of the Portugal 2020 framework, Regional OP Centro (POCI-01-0145-FEDER-030588), Regional OP Lisboa (Lisboa-010145-FEDER-03058) and by FCT/MEC through national funds, under Project PES3N (SAICT-45-2017-02) and Instituto de Telecomunicações project UID/EEA/50008/2013.
}

highly efficient, nonlinear amplifiers (such as the ones of class E, F [5] or D [6]) are combined to obtain an efficient amplification. The unavoidable losses associated with the combination process of the LINC can be compensated by the fact that we can employ efficient, grossly nonlinear power amplifiers, with each amplifier driven by a signal with a quarter of the required power that would be necessary if a single amplifier was employed.

In terms of monolithic implementation, namely using a low cost CMOS technology, the downscaling evolution of the technology nodes verified in the past decades has enabled the integration of an increasing number functionalities in the same integrated circuit (IC) die, in a system on chip (SoC) architecture [7]. However, this size downscaling is followed by a reduction of the maximum supply voltage $\left(V_{\text {supply }}\right)$ that can be applied to the IC [8], thus imposing serious limitations on the maximum output power (related with $V_{\text {supply }}^{2}$ ) that can be extracted from one single traditional RF Power Amplifier (namely, using a class A biasing scheme). To overcome it, parallel circuit techniques [9] can be used to combine the power of a set of multiple RF power amplifier branches operating, each one, at a low supply voltage.

In this work, we propose the multi-branch LINC (MBLINC) technique where $P$ nonlinear amplifiers are considered to achieve an efficient amplification and at the same contributing to improve the total output RF power. Therefore, the power consumption of each amplifier can be made very low by increasing $P$. One of the major problems of conventional LINC is the nonlinear distortion effects caused by the imbalances of the amplifiers' gain. In this work, it is shown that the robustness of MB-LINC to nonlinear distortion effects regarding mismatches in the amplifiers' gain is similar to that of conventional, two-branch LINC.

This work is organized as follows: in Sec. II a brief review of conventional LINC schemes is presented. Sec. III is dedicated to the explanation of the proposed MB-LINC amplification scheme. In Sec. IV it is shown how the nonlinear distortion effects associated to the MB-LINC can be evaluated and a set of results regarding the power spectral density (PSD) and the self-to-interference (SIR) of the resultant MB-LINC signals are presented. Sec. V shows the conclusions of this paper. 


\section{Conventional LiNC}

Let us start by considering a given bandpass signal written in the form

$$
s^{(b p)}(t)=r(t) \cos \left(2 \pi f_{c} t+\phi(t)\right),
$$

where $f_{c}$ represents the carrier frequency. Its complexenvelope, $s(t)$ can be written as

$$
s(t)=r(t) \exp (j \phi(t)),
$$

where $r(t)$ and $\phi(t)$ represent the absolute value and phase, respectively. In conventional LINC techniques, the signal $s(t)$ is separated into $N=2$ constant-envelope and out-phasing signals, $s_{1}(t)$ and $s_{2}(t)$. Without loss of generalization, we will illustrate the LINC concept for the specific case where the envelope is real-valued i.e., $\phi(t)=0$, which means that $s(t)=r(t)$ (the extension to other cases is straightforward).

Fig. 1 represents the conventional LINC decomposition under two components that lie in a circle with radius $R_{M} / 2$. From the vectorial representation of the figure, we can define

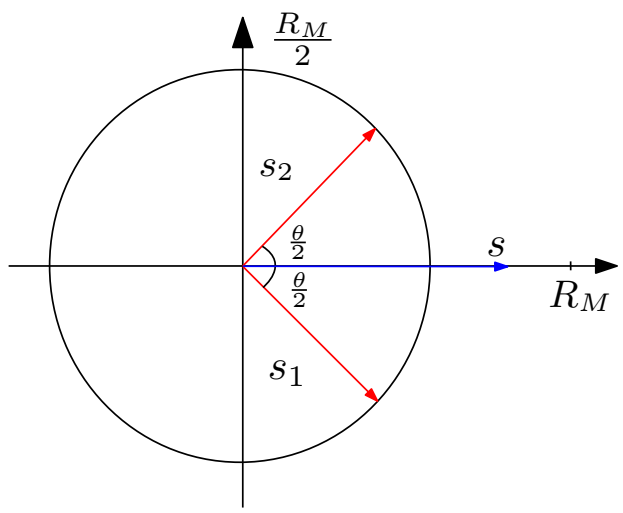

Fig. 1. Vectorial representation of the conventional LINC decomposition

the two LINC components as

$$
s_{1}(t)=\frac{R_{M}}{2} \exp \left(-j \frac{\theta(t)}{2}\right),
$$

and

$$
s_{2}(t)=\frac{R_{M}}{2} \exp \left(j \frac{\theta(t)}{2}\right) .
$$

Since these two signals have constant-envelope, they can be efficiently amplified with nonlinear power amplifiers. When the amplifiers' gain are equal, the combination of the two LINC components produce a scaled replica of $s(t)$, eventually clipped to a level $R_{M}$. In fact, the combination of the two signals can be generally thought as a clipped, nonlinearly distorted version of $s(t)$, since the maximum amplitude of the combined signal is limited to $R_{M}$ (which is a value obtained when $\theta / 2=0$ ). Under these conditions, the LINC process can be modeled as a bandpass memoryless nonlinearity characterized by the corresponding amplitude modulation/amplitude modulation (AM-AM) and amplitude modulation/phase modulation (AM-PM) conversion functions [10]. The complex envelope of the nonlinearly distorted signal can be written as

$$
y(t)=A(r(t)) \exp (j(\phi(t)+\Theta(r(t))),
$$

where $A(r(t))$ and $\Theta(r(t))$ represent the AM-AM and the AM-PM conversion functions, respectively. For the specific case of an envelope clipping, we have $\Theta(r(t))=0$ and

$$
A(r(t))= \begin{cases}r(t), & r(t) \leq R_{M} \\ R_{M}, & r(t)>R_{M}\end{cases}
$$

where $R_{M}$ denotes the clipping level. Still considering the geometry represented in Fig. 1, we can write, omitting the time dependence,

$$
s=\frac{R_{M}}{2} \exp \left(-j \frac{\theta}{2}\right)(1+\exp (j \theta))=R_{M} \cos \left(\frac{\theta}{2}\right) .
$$

Note also that

$$
\frac{\theta}{2}=\arccos \left(\frac{s}{R_{M}}\right) .
$$

Under these conditions, and since $\sin (\arccos (x))=$ $\sqrt{1-\cos ^{2}(\arccos (x))} \approx \sqrt{1-x^{2}}$, we can write

$$
s_{1}=\frac{s}{2}+j \frac{R_{M}}{2} \sqrt{1-\frac{s^{2}}{R_{M}^{2}}} \approx \frac{s}{2}+j \frac{1}{2} \sqrt{R_{M}^{2}-s^{2}},
$$

and

$$
s_{2}=\frac{s}{2}-j \frac{1}{2} \sqrt{R_{M}^{2}-s^{2}} .
$$

Besides this "global nonlinearity" of the LINC process, which is an envelop clipping, we can define a bandpass memoryless nonlinearity for the $p$ th branch (where $p$ can be either 1 or 2 , depending on the specific amplification branch), whose the AM/PM conversion function is defined as

$$
\Theta_{p}(r)=(-1)^{p} \arccos \left(\frac{r}{R_{M}}\right),
$$

and the $\mathrm{AM} / \mathrm{AM}$ conversion function is $A_{p}(r)=\frac{R_{M}}{2}$.

\section{Multi-BRAnCh LINC}

In the following, we propose the MB-LINC transmitter, which generalizes the LINC concept by considering up to $P$ amplification branches. In this technique, the signal $s(t)$ is divided into $P$ components that are amplified separately through a set of nonlinear amplifiers, as is shown. in Fig. 2. As in conventional LINC, when there are $P$ amplifying branches, the vectorial representation of the different LINC components is such that their sum produces the envelope at a given time instant.

Fig. 3 shows the vectorial representation of the MB-LINC components when $P$ is even $(P=4)$ and when $P$ is odd $(P=5)$. From this figure, it can be noted that, regardless of the value of $P$, the $p$ th signal (i.e., the signal associated with the $p$ th amplifying branch) of the MB-LINC decomposition is defined as

$$
s_{p}=\frac{R_{M}}{P} \exp \left(j \frac{\theta}{2}(2 p-P-1)\right) .
$$




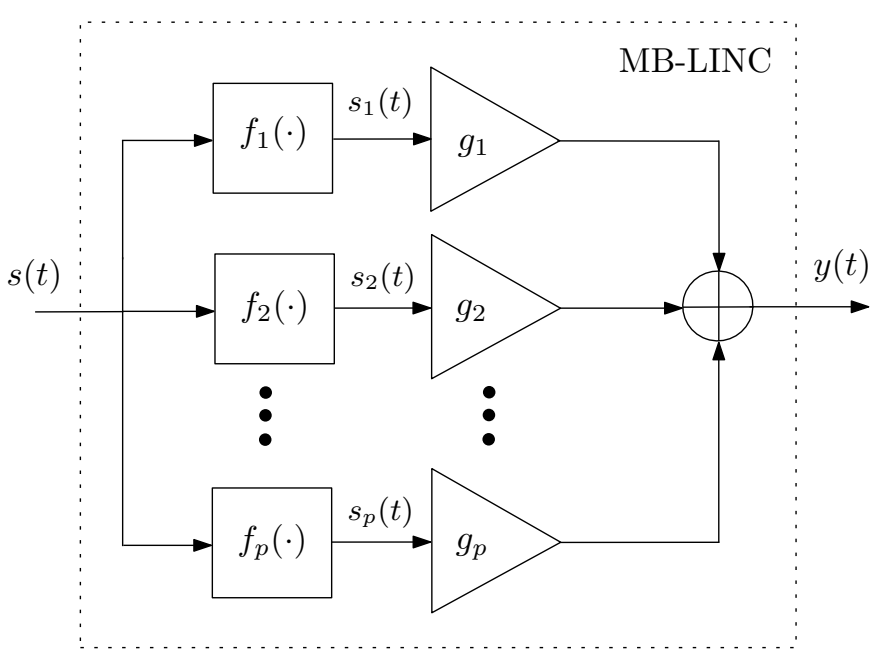

Fig. 2. Vectorial representation of the conventional LINC decomposition.
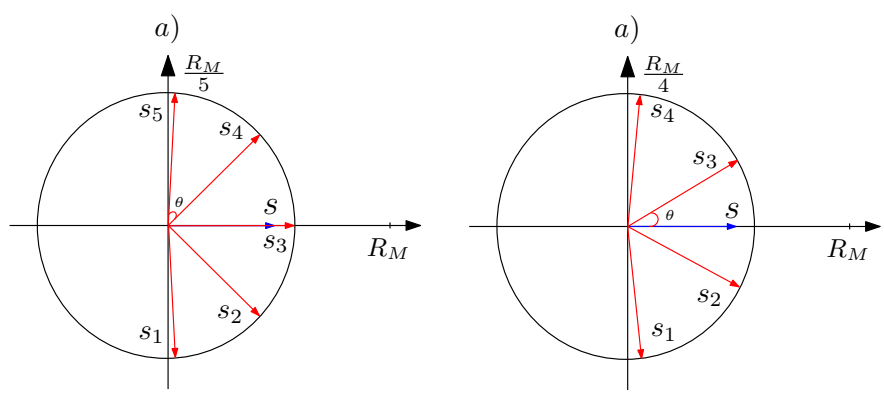

Fig. 3. Vectorial representation of the MB-LINC decomposition when $P=5$ (a) and when $P=4$ (b).

Therefore, the complex envelope of the MB-LINC output, given by the combination of the $P$ signals, can be written as

$$
\begin{aligned}
s & =\sum_{p=1}^{P} s_{p} \\
& =\sum_{p=1}^{P} \frac{R_{M}}{P} \exp \left(j \frac{\theta}{2}(2 p-P-1)\right) \\
& =\frac{R_{M}}{P} \exp \left(-j \frac{\theta}{2}(P-1)\right) \frac{1-\exp (j \theta P)}{1-\exp (j \theta)} .
\end{aligned}
$$

Under these conditions, we may write

$$
s=\frac{R_{M}}{P} \frac{\sin \left(P \frac{\theta}{2}\right)}{\sin \left(\frac{\theta}{2}\right)}=R_{M} f_{P}(\theta)
$$

with

$$
f_{P}(\theta)=\frac{\sin \left(P \frac{\theta}{2}\right)}{P \sin \left(\frac{\theta}{2}\right)}
$$

It should be noted that, if $P=2$, we have the conventional two branch LINC, i.e.,

$$
s=\frac{R_{M}}{2} \frac{2 \sin \left(\frac{\theta}{2}\right) \cos \left(\frac{\theta}{2}\right)}{\sin \left(\frac{\theta}{2}\right)}=R_{M} \cos \left(\frac{\theta}{2}\right),
$$

as obtained in (7).

Fig. 4 shows the envelope of a given OFDM signal before and after the LINC amplification scheme. The values of the envelope at the points $\mathrm{A}, \mathrm{B}$ and $\mathrm{C}$ can be vectorial built by the LINC components represented in the Fig. 5.

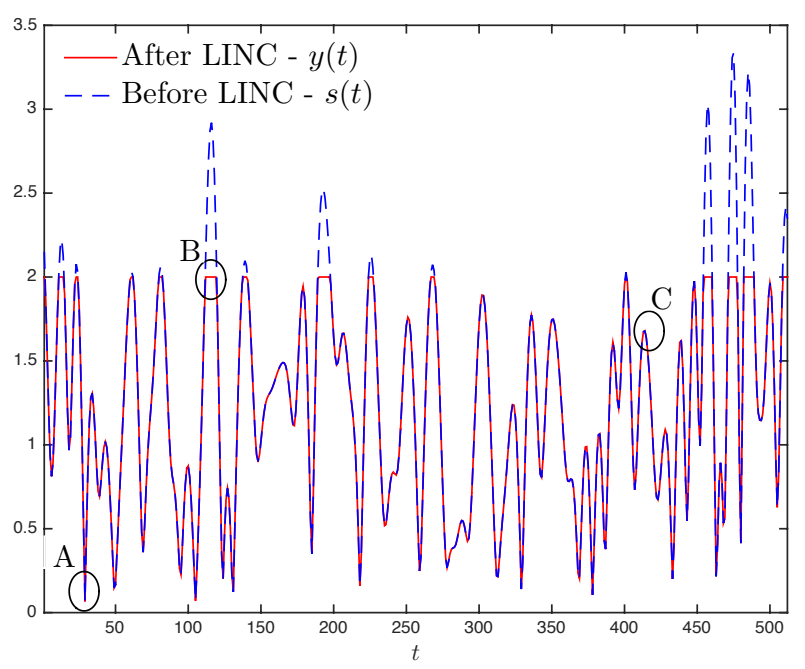

Fig. 4. Envelope of a given OFDM signal before and after the LINC amplification scheme.
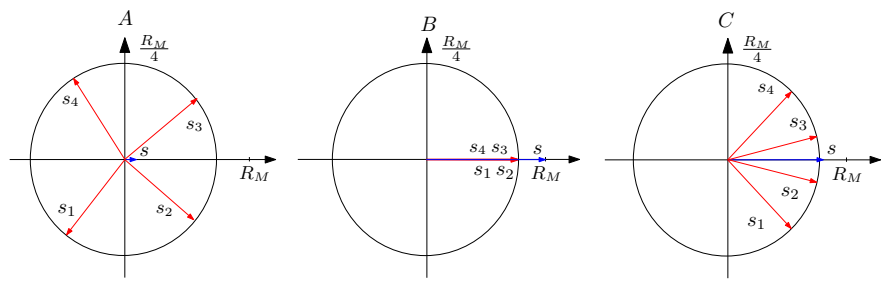

Fig. 5. Vectorial representation of the MB-LINC components with $P=4$ for the three envelope values identified in Fig. 5: almost 0 (A), clipped to $R_{M}$ (B) and lower than $R_{M}(\mathrm{C})$.

Clearly, when the envelope is almost 0 , the phase of the LINC components approaches $\pm \pi / 2$. On the other hand, when the envelope is clipped, the LINC components are aligned with the direction of $s$, yielding the maximum output value. The case $\mathrm{C}$ illustrates a situation where $s<R_{M}$.

In general, for a given $P, \theta$ is defined as

$$
\theta=f_{P}^{-1}\left(\frac{r}{R_{M}}\right) \text {. }
$$

Therefore, we can rewrite the output signal associated to the $p$ th branch as

$$
s_{p}=\frac{R_{M}}{P} \exp \left(j\left(f_{P}^{-1}\left(\frac{r}{R_{M}}\right) \frac{2 p-P-1}{2}\right)\right) .
$$

Thus, taking into account (12), one can define the AM/PM and $\mathrm{AM} / \mathrm{AM}$ conversion functions associated to the $p$ th branch as

$$
\Theta_{p}(r)=f_{P}^{-1}\left(\frac{r}{R_{M}}\right) \frac{2 p-P-1}{2},
$$


and

$$
A_{p}(r)=\frac{R_{M}}{P} .
$$

It is well-known that imbalances in the complex gains of LINC's amplifiers lead to performance degradation [12], [13]. In that context, one could expect the situation to be more serious with MB-LINC, since there are more amplifying branches. To study the impact of these imbalances on the MB-LINC signal, let us consider the combined signal after amplification, which is given by

$$
s=\sum_{p=1}^{P} g_{p} \frac{R_{M}}{P} \exp \left(j\left(f_{P}^{-1}\left(\frac{r}{R_{M}}\right) \frac{2 p-P-1}{2}\right)\right)
$$

where $g_{p}$ is the complex gain associated with the $p$ th amplifier, defined as $g_{p}=1+\varepsilon_{g}$, with $\varepsilon_{g}$ being a complex-valued deviation whose the real and imaginary parts have variance $\sigma_{\varepsilon}^{2}$, i.e., $\varepsilon_{g} \sim \mathcal{C N}\left(0,2 \sigma_{\varepsilon}^{2}\right)$. Naturally, when $\sigma_{\varepsilon}=0$, the amplifiers have equal gains and (21) turns into (16). On the other hand, when $\sigma_{\varepsilon}>0$, the magnitude of nonlinear distortion effects associated with the MB-LINC signal will depend on the combination of the gains of the different amplifiers.

\section{Nonlinear Distortion EFFECTS}

By taking advantage of the Gaussian nature of OFDM signals with a large number of subcarriers $N$, the PSD of their nonlinearly distorted version can be obtained through their autocorrelation, $R_{y}(n)$, that is itself a function of the input signals' autocorrelation, $R_{s}(n)$, and of the power associated to the different intermodulation products (IMPs) at the nonlinearity output. The autocorrelation of a nonlinearly distorted OFDM signal can be computed as [14]

$$
R_{y}(n)=\sum_{\gamma=0}^{+\infty} P_{2 \gamma+1} \frac{R_{s}(n)^{\gamma+1}\left(R_{s}^{*}(n)\right)^{\gamma}}{\sigma^{2(2 \gamma+1)}},
$$

where $\sigma$ is the standard deviation of the input signal and $P_{2 \gamma+1}$ is the power associated with the IMP of order $2 \gamma+1$. The PSD of the nonlinearly distorted signal, $G_{y}(k)$, is given by $\operatorname{DFT}\left(R_{y}(n)\right)$. Moreover, from the Bussgang's theorem [15], we can express the nonlinearly distorted signal as the sum of two components. Under these conditions, the PSD of MBLINC signals can be written as

$$
G_{y}(k)=|\alpha|^{2} G_{s}(k)+G_{d}(k),
$$

where $\alpha$ is a scale factor given by the scaled cross-correlation between the OFDM signal and its nonlinear distorted version, and $G_{s}(k)$ and $G_{d}(k)$ are the PSDs of the input signal and nonlinear distortion term, respectively.

Fig. 6 shows the normalized PSD of the nonlinear distortion term, $g_{d}(k)=G_{d}(k) / \max \left(|\alpha|^{2} G_{s}(k)\right)$, considering different values of $P, \sigma_{\varepsilon}=0$ and $R_{M} / \sigma=1.5$ (due to the symmetry associated to the different amplification branches, only $(P-$ 1) $/ 2+1$ PSDs are shown). From the figure, it can be noted that although the PSD of the nonlinear distortion term associated to the different branches can vary, the PSD associated to the combined signal is the same, regardless of $P$. To have an
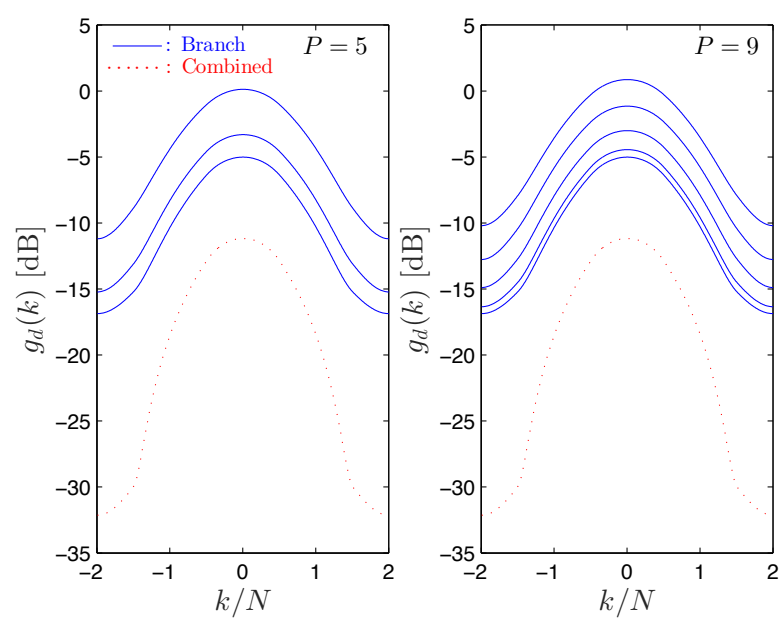

Fig. 6. Normalized PSD of the nonlinear distortion term associated to the different MB-LINC branches.

insight on the performance penalty associated to the nonlinear distortion, we can define the SIR for a the $k$ th subcarrier as

$$
\operatorname{SIR}_{k}=\frac{|\alpha|^{2} G_{s}(k)}{G_{d}(k)} \text {. }
$$

Fig. 7 shows the evolution of $\operatorname{SIR}_{k}$ considering an MB-LINC transmitter with $s_{M} / \sigma=1.0$, different values of $P$ and $\sigma_{\varepsilon}=0.05$. From the figure, it can be noted that although
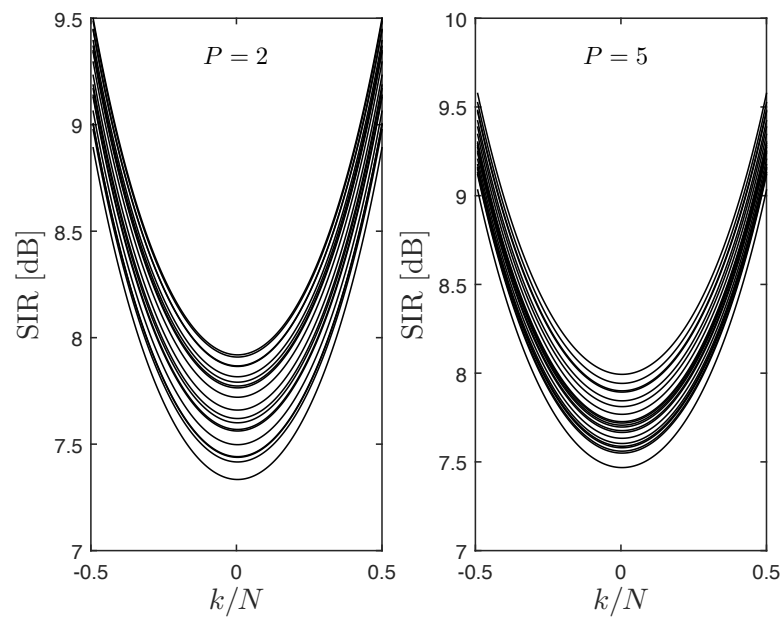

Fig. 7. Evolution of $\operatorname{SIR}_{k}$ considering an MB-LINC transmitter with different values of $P$ and $\sigma_{\varepsilon}=0.05$.

there are variations on the SIR for different gain combinations, the magnitude of these variations is similar. Therefore, the use $P>2$ does not increase the nonlinear distortion effects comparatively to the conventional LINC, where $P=2$.

\section{CONCLUSIONS}

In this work we propose the MB-LINC technique, which generalizes the conventional LINC. It is shown that, although 
each amplifier can work with low power signals, the spectral characteristics of the combined signal does not change relatively to the conventional LINC techniques, even when there are imbalances in the amplifiers' gain.

\section{REFERENCES}

[1] L. Cimini, "Analysis and simulation of a digital mobile channel using orthogonal frequency division multiplexing," IEEE Trans. Commun., vol. 33, no. 7, pp. 665-675, Aug. 1985.

[2] Y. Rahmatallah and S. Mohan, "Peak-to-average power ratio reduction in OFDM systems: a survey and taxonomy", IEEE Communication Surveys and Tutorials, vol. 15, no. 4, pp. 1567-1592, Mar. 2013.

[3] J. Han and H. Lee, "An overview of peak-to-average power ratio reduction techniques for multicarrier transmission", IEEE Wireless Commun., vol. 12, no. 2, pp. 56-65, Apr. 2005.

[4] D. Cox, 'Linear amplification with nonlinear components,' IEEE Trans. Commun., vol. 22, no. 12, pp. 1942-1945, Dec. 1974.

[5] A. Banerjee, R. Hezar, L. Ding, N. Schemm and B. Haroun, "A $29.5 \mathrm{dBm}$ class-E outphasing RF power amplifier with performance enhancement circuits in 45nm CMOS,“ 40th IEEE European Solid State Circuits Conference, Venice, pp. 467-470, Sept. 2014.

[6] H. Xu, Y. Palaskas, A. Ravi, M. Sajadieh, M. A. El-Tanani and K. Soumyanath,"A Flip-Chip-Packaged $25.3 \mathrm{dBm}$ Class-D Outphasing Power Amplifier in $32 \mathrm{~nm}$ CMOS for WLAN Application," IEEE Journal of Solid-State Circuits, vol. 46, no. 7, pp. 1596-1605, Jul. 2011.

[7] B. Francois and P. Reynaert:"A Fully Integrated Watt-Level Linear 900$\mathrm{MHz}$ CMOS RF Power Amplifier for LTE-Applications, "IEEE Trans. on Microwave Theory and Techniques, vol. 60, no. 6, pp. 1878-1885, June 2012.

[8] A. Banerjee, R. Hezar and L. Ding,"Efficiency improvement techniques for RF power amplifiers in deep submicron CMOS," IEEE Custom Integrated Circuits Conference, San Jose, CA, Sept. 2015.

[9] S. Kang, D. Baek and S. Hong,"A 5-GHz WLAN RF CMOS Power Amplifier With a Parallel-Cascoded Configuration and an Active Feedback Linearizer," IEEE Trans. on Microwave Theory and Techniques, vol. 65, no. 9, pp. 3230-3244, Sept. 2017.

[10] A. Kaye, D. George and M. Eric, "Analysis and compensation of bandpass nonlinearities for communications," IEEE Trans. Commun., vol. 20, no. 5, pp. 965-972, Oct. 1972.

[11] R. Dinis, and A. Gusmão, "Nonlinear signal processing schemes for OFDM modulations within conventional or LINC transmitter structures,' European Trans. on Telecomm., vol. 19, no. 3, pp. 257-271, Jan. 2008.

[12] J. Guerreiro, R. Dinis and P. Montezuma, "A simple method for the analytical characterization of OFDM schemes with LINC transmitter structures," ISWCS'15, Brussels, Belgium, Aug. 2015.

[13] F. Casadevall, and A. Valdovinos "Performance analysis of QAM modulations applied to the LINC transmitter," IEEE Trans. Veh. Technol., vol. 42, no. 4, pp. 399-406, Nov. 1993.

[14] R. Dinis and A. Gusmão, "A class of nonlinear signal processing schemes for bandwidth-efficient OFDM transmission with low envelope fluctuation," IEEE Trans. Commun., vol. 52, no. 11, pp. 2009-2018, Nov. 2004

[15] H. Rowe: "Memoryless nonlinearities with Gaussian input: elementary results," Bell System Tech. Journal, vol. 61, no. 7, pp. 1519-1526, Sept. 1982. 